\title{
POTENCIAL DE PRODUCCIÓN DE LÍNEAS DE FRIJOL ADZUKI (Vigna angularis), EN TRES LOCALIDADES DE COSTA RICA*
}

\author{
Rodolfo Araya**, Edy Valerio*, Hugo Hernández***
}

\begin{abstract}
RESUMEN
En tres localidades de Costa Rica: Alajuela a $840 \mathrm{msnm}$, Pérez Zeledón a 760 msnm y Río Frío a 100 msnm, se evaluaron 14 líneas de frijol Adzuki para grano seco, durante el periodo 1984 a 1988. Como testigos se emplearon tres variedades de frijol común: Talamanca, Huetar y México 80.

Las mejores líneas de Adzuki, en las tres localidades, fueron la UCR 2 y UCR 7 de grano color rojo y la UCR 3 de grano de color negro. El Adzuki en Río Frío y Pérez Zeledón, mostró una produc-ción similar a la del frijol común debido a la baja incidencia de Mustia hilachosa (Thanatephorus cucumeris), hongo que afecta poco al Adzuki, pero que es el principal limitante a la producción de frijol común en estas dos zonas. En Alajuela, zona apropiada para un buen desarrollo de frijol común, varias líneas de Adzuki mostraron una mayor productividad.
\end{abstract}

\begin{abstract}
Production potential of adzuki bean (Vigna angularis) lines in three localities in Costa Rica. Between 1984 and 1988, fourteen adzuki bean lines were evaluated in three localities in Costa Rica: Alajuela at 840 masl., Perez Zeledon at 760 masl. and Rio Frio at 100 masl. Three common bean varieties were used as controls: Talamanca, Huetar and Mexico 80. The best adzuki lines in the three localities were U CR - 2 and U CR - 7 (red colored grain) and UCR-3 (black colored grain). In Rio Frio and Perez Zeledon, the adzuki showed a like production as the common bean, due to the low incidence of Web blight (Thanatephorus cucumeris Frank Donk). This is a fungus which slightly affects the adzuki bean, but it is a limiting factor for common bean production in these two localities. In Alajuela, an ideal zone for common bean production, several adzuki lines showed a higher productivity.
\end{abstract}

\section{INTRODUCCIÓN}

El frijol Adzuki (Vigna angularis (Wild) Ohwi \& Ohashi (1969)), fue clasificado antiguamente como Phaseolus angularis (Wild) W. F. Wight (1909), (CIAT 1980) sinonimia Phaseolus mungo L. var. angularis (Mateo Box 1961); es conocido como judía Adzuki; Adzuki Bean, Diamante rojo, soja chincsa y feijao japones (Mateo Box 1961; Vieira 1967).

Esta leguminosa es originaria del Centro y oeste de la China (Centro I de Vavilov). En este país se le utiliza como planta medicinal para tratar tumores, abscesos, molestias del riñón, dificultades en parto, retención de placenta y estreñimiento (Duke 1981; Sacks 1977). Las semillas cocidas son utilizadas en la alimentación humana, tanto solas como mezcladas con arroz.

También se consumen sus granos pregerminados. En Japón es el segundo grano más utilizado después de la soya. Se emplea también esta leguminosa como cultivo forrajero o de abono verde (Andrade 1975; Sacks 1977). Esta leguminosa es más apropiada para zonas calientes y de lluvias persistentes, debido a su resistencia a la telaraña (Thanatephorus cucumeris) (Delgado y Araya 1983) pero también se le indica como tolerante a la sequía (Centre for Oversea Pest-Research 1981).

La variabilidad genotípica es amplia en frijol Adzuki, existen mas de 60 variedades que difieren en su ciclo

\footnotetext{
* Trabajo presentado a la XXXVIII Reunión Anual del PCCMCA, Managua, Nicaragua, 23-27 de marzo de 1992.

** Programa de Leguminosas de Grano. Estación Experimental Fabio Baudrit Moreno, Apartado 183, 4050 Alajuela, Costa Rica.

***Finca Experimental Río Frío. Facultad de Agronomía, Universidad de Costa Rica, San José, Costa Rica.
} 
vegetativo, color y tamaño de las semillas y sensibilidad a la longitud del día, aunque se indica como planta de día corto (Duke 1981, Mateo Box 1961).

El potencial de producción de grano en Costa Rica ha variado de 567 a $1800 \mathrm{~kg} / \mathrm{ha}$ (Delgado Araya 1983; Escalante et al. 1985, Universidad de Costa Rica 1986) pero se indica hasta $3700 \mathrm{~kg} / \mathrm{ha}$ (Robinson 1980) en Minnessota, U.SA., con la variedad Minoka.

La densidad de siembra para esta leguminosa es similar a la indicada para frijol común (Phaseolus vulgaris L.) de hábito de crecimiento arbustivo, tipo II, con un promedio de 250,000 plantas/ha (Lawn 1983; Delgado y Araya 1983; Escalante et al. 1985).

En asocio con camote (Ipomoea batata L.), esta leguminosa produjo la menor reducción de la producción de este tubérculo (Escalante et al. 1985), y puede ser una leguminosa apropiada para asociar con maíz en siembra simultánea debido a su reducido ciclo vegetativo, hábito arbustivo y de bajo porte, condiciones en las cuales el frijol común asociado a maíz no ha afectado su producción y en ciertas condiciones lo ha incrementado (Araya y Zumbado 1983; González et al. 1984).

El objetivo de esta investigación fue evaluar la productividad de líneas de frijol Adzuki en comparación con variedades comerciales de frijol común.

\section{MATERIALES Y MÉTODOS}

El estudio se efectuó en tres localidades de Costa Rica: Estación Experimental Fabio Baudrit Moreno, ubicada en la provincia de Alajuela a $840 \mathrm{msnm}$, en el Cajón de Pérez Zeledón, provincia de San José a 760 msnm y en la Finca Experimental Río Frío ubicada en la provincia de Heredia a $100 \mathrm{msnm}$.

El periódo experimental estuvo comprendido del 18 de mayo al 18 de diciembre de 1986 y del 22 de setiembre al 14 de diciembre de 1987 en Alajuela, del 28 mayo al 18 de agosto de 1988 en Pérez Zeledón y del 17 de noviembre de 1984 al 31 de enero de 1985 y del 20 de noviembre de 1985 al 20 de febrero de 1986 en Río Frío.
Las líneas de frijol Adzuki y las variedades de frijol común evaluadas, se incluyen en el Cuadro 1.

Cuadro 1. Líneas de frijol adzuki y variedades de frijol común evaluadas en tres localidades 1985-1988.

\begin{tabular}{lcll}
\hline Líneas* & $\begin{array}{l}\text { Código de } \\
\text { Procedencia }\end{array}$ & Origen & $\begin{array}{l}\text { Color } \\
\text { grano }\end{array}$ \\
\hline UCR-1 & ---- & ---- & rojo \\
UCR-2 & 416742 & Japón & rojo \\
UCR-3 & 157626 & Korea & negro \\
UCR-4 & 221974 & Japón & rojo \\
UCR-5 & 360701 & Taiwán & rojo \\
UCR-6 & 157625 & Korea & rojo \\
UCR-7 & 358735 & Japón & rojo \\
UCR-8 & 330587 & Belgium & rojo \\
UCR-9 & 416744 & Japón & rojo \\
UCR-10 & 157643 & Korea & rojo \\
UCR-11 & 273486 & Korea & rojo \\
UCR-12 & 157649 & Korea & rojo \\
UCR-13 & 196173 & Korea & rojo \\
UCR-14 & 330588 & Belgica & rojo \\
Huetar*** & --- & Costa Rica & rojo \\
Talamanca*** & ---- & CIAT & negro \\
México 80*** & ---- & Costa Rica & rojo \\
\hline
\end{tabular}

*Código del Banco de Germoplasma de La Estación Experimental Fabio Baudrit M.

**Código del U.S. Department of Agriculture. Agricultural

Research Service. Plant Science, Research Division. $\star * *$ Variedades comerciales de frijol común en costa Rica, hábito crecimiento IIb.

En Alajuela y Pérez Zeledón se utilizó un diseño de bloques completos al azar con cuatro repeticiones. La unidad experimental constó de cuatro hileras de $4 \mathrm{~m}$ de largo distanciadas a $0,5 \mathrm{~m}$ y a $0,08 \mathrm{~m}$ entre plantas. En ambos ensayos la distancia entre plantas fue de $0,5 \mathrm{~cm}$, el área útil de la parcela experimental fueron las dos hileras centrales excluyendo una planta de cada uno de los extremos de las hileras.

En Río Frío se utilizó el mismo diseño experimental pero con tres repeticiones. La parcela experimental estuvo formada por tres hileras de $3 \mathrm{~m}$ de largo espaciadas a 0,6 $\mathrm{m}$. El área útil de estas parcelas fue el surco central para el primer ensayo 1984-1985. En el segundo ensayo 19851986, la parcela estuvo formada por cinco hileras de $4 \mathrm{~m}$ de largo separadas $0,5 \mathrm{~m}$ entre sí, la parcela útil fueron las tres hileras centrales y sólo se eliminaron las dos primeras plantas de los extremos de cada hilera.

Las variables evaluadas fueron: Rendimiento en grano, número de vainas por planta, con base en todas las 
plantas de una hilera de la parcela útil, número de granos por vaina, con base en todas las vainas de las plantas de una hilera de la parcela útil; peso de 100 granos, con base en el peso promedio de todos los granos obtenidos en una hilera de la parcela útil. En los dos primeros ensayos efectuados en Alajuela solo se obtuvo el rendimiento en grano.

La preparación del terreno se efectuó mediante una arada una rastreada de los terrenos y luego con su surcador se elaboraron camellones. El combate de malezas se efectuó con la aplicación en prebrotación de pendimetalina (Prowl) y DNBP (Herbón) en dosis de 2,25 1/ha y 41 /ha respectivamente.

A la siembra y al fondo del surco se adicionó fertilizante de fórmula comercial 10-30-10 a razón de $33,3-43,7-27,7 \mathrm{~kg} / \mathrm{ha}$ de NPK, respectivamente.

\section{RESULTADOS}

Hubo diferencias significativas, entre las lineas de adzuki y variedades de frijol común evaluadas, en todas las localidades y épocas de siembra, Cuadros 2, 3, 4, 5 y 6.

La producción del frijol común fue superior o similar a la obtenida con las lineas de frijol adzuki, inclusive en la localidad de Rio Frio. En esta zona por lo general hay alta incidencia de telaraña (Thanetephorus cucumeris), para lo cual sólo el frijol adzuki posee una mayor resistencia, pero durante los dos ensayos efectuados el frijol común no se afectó por este hongo debido posiblemente a la baja presión de inóculo, ya que la lluvia durante los meses de las investigaciones no fue apropiada para el desarrollo de esta enfermedad (primer ensayo: 343,316 y $94 \mathrm{~mm}$ para noviembre, diciembre 1984 y enero de 1985, respectivamente y para el segundo ensayo de 395, 221, 28, $228 \mathrm{~mm}$, para diciembre 1985 y enero, febrero y marzo de 1986, respectivamente.

En caso de siembras continuas de frijol común en estos terrenos si se podría esperar severos daños por telaraña dada las condiciones de alta precipitación y humedad relativa, así como temperaturas superiores a $24^{\circ} \mathrm{C}$.

Cuadro 2. Producción de grano en frijol Adzuki y frijol común durante dos épocas de siembra en Alajuela, Costa Rica, 1986.

\begin{tabular}{lll}
\hline & \multicolumn{2}{c}{ PRODUCCION $(\mathrm{kg} / \mathrm{ha})$} \\
\cline { 2 - 3 } Linea o variedad & Mayo & Setiembre \\
\hline UCR 1 & $1262 \mathrm{c}$ & $1323 \mathrm{c}$ \\
UCR 2 & $1325 \mathrm{~b}$ & $1333 \mathrm{c}$ \\
UCR 3 & $1446 \mathrm{ab}$ & $1319 \mathrm{c}$ \\
UCR 4 & $1571 \mathrm{a}$ & $1417 \mathrm{bc}$ \\
UCR 5 & $1682 \mathrm{a}$ & $1576 \mathrm{ab}$ \\
UCR 6 & $1117 \mathrm{c}$ & $1052 \mathrm{~d}$ \\
UCR 7 & $1594 \mathrm{a}$ & $1465 \mathrm{bc}$ \\
UCR 8 & $1047 \mathrm{~cd}$ & $1382 \mathrm{bc}$ \\
UCR 9 & $1259 \mathrm{c}$ & $1323 \mathrm{c}$ \\
UCR 10 & $1354 \mathrm{~b}$ & $1059 \mathrm{~d}$ \\
UCR 11 & $1164 \mathrm{c}$ & $1431 \mathrm{bc}$ \\
UCR 12 & $1659 \mathrm{a}$ & $1330 \mathrm{c}$ \\
UCR 13 & $1665 \mathrm{a}$ & $1538 \mathrm{~b}$ \\
UCR 14 & $1227 \mathrm{c}$ & $1288 \mathrm{c}$ \\
HUetar & $1278 \mathrm{c}$ & $1764 \mathrm{a}$ \\
Talamanca & $1269 \mathrm{c}$ & $1733 \mathrm{a}$ \\
\hline
\end{tabular}

Cuadro 3. Producción de grano y otras variables agronómicas evaluadas en líneas de frijol Adzuki en Alajuela, Costa Rica, 1987.

\begin{tabular}{|c|c|c|c|c|c|c|c|c|c|}
\hline \multirow{2}{*}{$\begin{array}{l}\text { Línea o } \\
\text { Variedad } \\
\text { UCR-1 }\end{array}$} & \multicolumn{2}{|c|}{$\begin{array}{l}\text { Producción } \\
\quad \text { (kg/ha) }\end{array}$} & \multirow{2}{*}{$\begin{array}{l}\begin{array}{l}\text { Vainas/ } \\
\text { planta }\end{array} \\
6,4 \text { abcd }\end{array}$} & \multicolumn{2}{|c|}{$\begin{array}{c}\text { Granos/ } \\
\text { vaina }\end{array}$} & \multicolumn{2}{|c|}{$\begin{array}{l}\text { Peso } 100 \\
\text { granos }(\mathrm{g})\end{array}$} & \multicolumn{2}{|c|}{$\begin{array}{l}\text { Altura de } \\
\text { planta }(\mathrm{cm})\end{array}$} \\
\hline & 1708 & def & & 6,1 & $a b$ & 10,6 & $\mathrm{~h}$ & 34,0 & cde \\
\hline CUR-2 & 2132 & bcde & 6,5 abcd & 5,5 & $a b c$ & 11,3 & gh & 31,3 & efg \\
\hline UCR-3 & 2790 & $a b c$ & 9,9 a & 4,5 & de & 15,2 & $d$ & 33,5 & deg \\
\hline UCR-4 & 2385 & bcd & 6,9 abcd & 5,7 & $a b c$ & 13,5 & e & 39,5 & bc \\
\hline UCR-5 & 2453 & bcd & 7,2 abcd & 5,7 & $a b c$ & 12,9 & efg & 44,5 & $b$ \\
\hline UCR-6 & 2099 & bcde & 8,5 abc & 4,0 & e & 12,7 & efg & 26,8 & $g$ \\
\hline UCR-7 & 936 & $f$ & $4,5 \quad d$ & 4,1 & $\mathrm{e}$ & 16,3 & $d$ & 27,8 & $f g$ \\
\hline UCR-8 & 1879 & cdef & 6,1 abcd & 6,2 & $a$ & 13,5 & $\mathrm{e}$ & 39,5 & bc \\
\hline UCR-9 & 1471 & def & $6,4 \mathrm{abcd}$ & 5,5 & $a b c$ & 11,6 & fgh & 30,3 & defg \\
\hline UCR-10 & 1575 & def & 5,1 & 5,5 & $a b c$ & 13,2 & ef & 28,5 & efg \\
\hline UCR-11 & 1777 & def & 6,2 abcd & 5,1 & $\mathrm{~cd}$ & 10,9 & h & 32,5 & efg \\
\hline UCR-12 & 1292 & ef & $5,0 \quad \mathrm{~cd}$ & 4,1 & $\mathrm{e}$ & 17,9 & c & 31,3 & efg \\
\hline UCR-13 & 2967 & $a b$ & 10,1 a & 4,1 & $\mathrm{e}$ & 18,3 & bc & 31,0 & efg \\
\hline UCR-14 & 2422 & bcd & 7,8 abcd & 6,2 & a & 12,0 & efgh & 38,5 & $c d$ \\
\hline Huetar & 2116 & bcde & 9,5 ab & 5,5 & $a b c$ & 19,8 & $b$ & 51,8 & a \\
\hline Talamanca & 3434 & $a$ & 5,6 bcd & 5,4 & bc & 22,1 a & & 52,5 & a \\
\hline
\end{tabular}


Cuadro 4. Producción de grano en frijol adzuki y frijol común en Río Frío, Heredia, Costa Rica Noviembre 1985-Enero 1986.

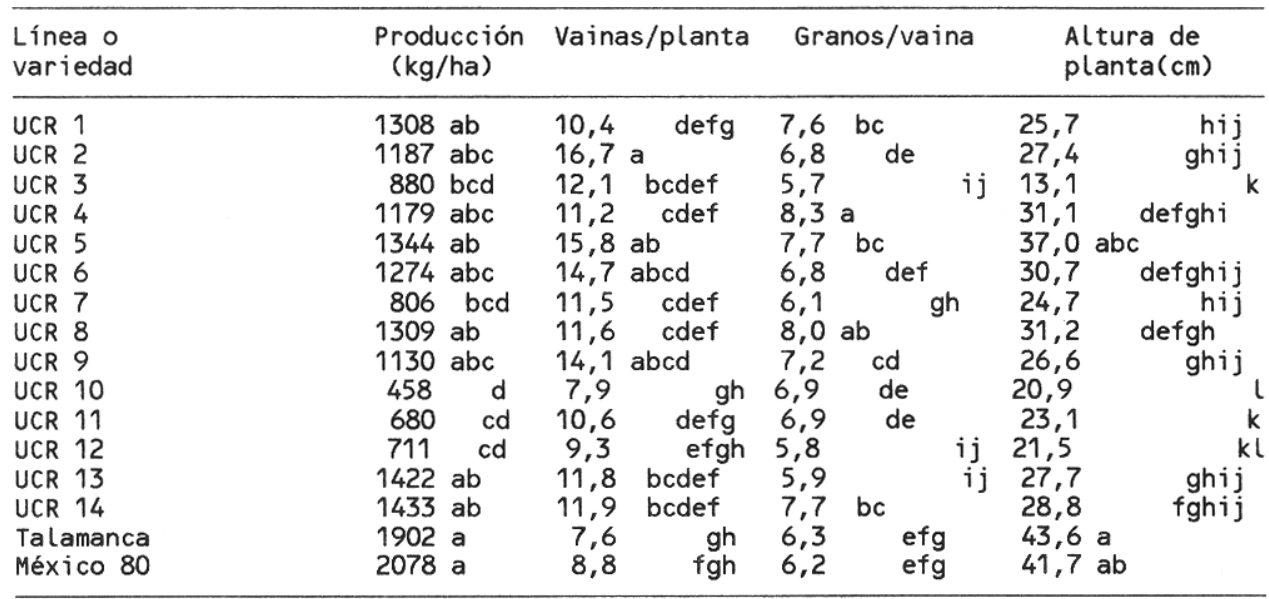

En el segundo ensayo efectuado en Río Frío se redujo la distancia de siembra entre hileras de 0,6 a $0,5 \mathrm{~m}$, pero se mantuvo el mismo número de granos por metro lineal de hilera, lo que incrementó en cerca de 66,000 semillas la densidad de siembra. El frijol adzuki mostró una respuesta positiva a esta mayor densidad con una mayor productividad, lo cual no sucedió con el rendimiento del frijol común, Cuadro 5 y 6 . Al respecto Hernández y Araya, 1986 obtuvieron la mayor producción de grano a $0,40 \mathrm{~m}$ de distancia entre hileras, pero con una población de 416,666 plantas/ha.

El frijol adzuki estuvo de cosecha entre los 60 y 65 días después de la siembra, pero el frijol común varió entre 70 días los mas precoces Mexico 80R y Huetar, hasta 85 días el Talamanca. Esto permite al frijol adzuki un mayor escape a enfermedades, por menor exposición al medio ambiente, ademas posee una arquitectura arbustiva, baja altura de planta y un tallo mas fuerte que influye en una ausencia casi total de volcamiento de planta, situación contraria a la observada en frijol comun y que debe de considerarse en esta zona que presenta fuertes vientos. Otra característica importante fue la incidencia de Babosas (Vaginulus plebeius) que se dió con menor intensidad en el Adzuki, pero en frijol común produjo daños aun en estados avanzados de desarrollo vegetativo.

En Río Frío la investigación efectuada con la línea UCR-1 de Adzuki y frijol criollo, evidenció la superioridad del adzuki en producción y baja incidencia de telaraña (Universidad de Costa Rica 1986). El potencial de esta línea fue superada, en estas evaluaciones por otras de mayor estabilidad como la UCR-7 UCR-2 y UCR-3 en esta zona.
En Alajuela el frijol común de mayor ciclo vegetativo (variedad Talamanca) mostró mayor productividad que el adzuki. La variedad Huetar solo mostró mayor rendimiento en un ensayo efectuado en setiembre de 1986 y poca diferencia en relación a la línea de adzuki mas productiva VCR-5, Cuadros 2 y 3.

En Pérez Zeledón el frijol común también superó al frijol adzuki pero bajo una situación similar a la observada en Alajuela. En esta localidad se presenta por lo regular gran incidencia de Telaraña, lo que no sucedió durante la presente investigación debido a la baja precipitación ocurrida, y por haberse efectuado la siembra en una área anteriormente sembrada con pastos, lo que influyó en una baja presión de inóculo.

Las líneas UCR 5, UCR 7, y UCR 13 fueron las mas promisorias en todas las localidades y épocas de siembra con base en su producción de grano, así como mayor producción de vainas, en la mayoría de los ensayos.

El frijol adzuki poseé un grano pequeño si se compara con el del frijol común que lo supera, en las variedades comerciales de Costa Rica, hasta en $10 \mathrm{~g} / 100$ semillas, y una altura de planta muy baja, (hasta $15 \mathrm{~cm}$ menor que la del frijol común), pero su potencial de producción es alto si se considera su reducido ciclo vegetativo, y muy apropiado para zonas de alta temperatura y alta precipitación (Delgado et al. 1983), su única desventaja esta en su sabor amargo en comparación con el frijol común. Esta característica de sabor puede ser mejorada con una investigación sobre la eliminación y neutralización de los componentes de la semilla que dan el "sabor amargo" . 
Cuadro 5. Producción de grano y otras variables agronómicas evaluadas en líneas de frijol Adzuki, en Pérez Zeledón,Costa Rica 1988.

\begin{tabular}{|c|c|c|c|c|c|c|}
\hline \multirow{2}{*}{$\begin{array}{l}\text { Línea o } \\
\text { Variedad } \\
\text { UCR-1 }\end{array}$} & \multirow{2}{*}{$\begin{array}{c}\begin{array}{c}\text { Producción } \\
(\mathrm{kg} / \mathrm{ha})\end{array} \\
1848 \mathrm{ab}\end{array}$} & \multirow{2}{*}{$\begin{array}{c}\begin{array}{c}\text { Vainas/ } \\
\text { planta }\end{array} \\
10,5 \text { abcd }\end{array}$} & $\begin{array}{c}\text { Granos/ } \\
\text { vaina }\end{array}$ & \multicolumn{2}{|c|}{$\begin{array}{l}\text { Peso } 100 \\
\text { granos }(\mathrm{g})\end{array}$} & \multirow{2}{*}{$\begin{array}{c}\begin{array}{c}\text { Altura de } \\
\text { planta }(\mathrm{cm})\end{array} \\
33,5\end{array}$} \\
\hline & & & 6,1 a & 8,3 & $f g$ & \\
\hline UCR-2 & $1247 \mathrm{ab}$ & $9,6 \mathrm{abcd}$ & $5,5 a b c$ & 7,9 & $\mathrm{~g}$ & 30,5 \\
\hline UCR-3 & $1201 \mathrm{ab}$ & 7,8 abcd & 4,5 de & 11,7 & & 31,5 \\
\hline UCR-4 & $1284 a b$ & $7,8 \mathrm{abcd}$ & $5,7 a b c$ & 10,9 & de & 38,5 \\
\hline UCR -5 & $1519 \mathrm{ab}$ & $11,0 \mathrm{abc}$ & $5,7 \mathrm{abc}$ & 8,7 & $\mathrm{fg}$ & 37,5 \\
\hline UCR-6 & $1012 \mathrm{~b}$ & $10,0 \mathrm{abc}$ & 4,0 & 9,7 & ef & 25,5 \\
\hline UCR-7 & $1709 a b$ & 11,0 abc & 4,1 & 12,5 & d & 30,0 \\
\hline UCR-8 & $1632 a b$ & $7,6 \mathrm{bcd}$ & 6,2 a & 9,1 & $\mathrm{fg}$ & 39,0 \\
\hline UCR-9 & $1403 a b$ & 9,4 abcd & $5,5 a b c$ & 8,2 & $f g$ & 31,0 \\
\hline UCR-10 & $1178 \mathrm{ab}$ & 8,1 abcd & $5,5 a b c$ & 9,3 & $\mathrm{fg}$ & 25,5 \\
\hline UCR-11 & $1413 a b$ & $11,7 \mathrm{ab}$ & 5,1 & 8,5 & $f g$ & 34,0 \\
\hline UCR-12 & $1822 a b$ & 9,9 abcd & 4,1 & 14,0 & c & 30,5 \\
\hline UCR-13 & $1989 \mathrm{ab}$ & 12,4 a & 4,1 & 14,4 & c & 30,5 \\
\hline UCR-14 & $1090 a b$ & $6,8 \mathrm{~cd}$ & 6,2 a & 8,0 & $\mathrm{fg}$ & 36,5 \\
\hline Huetar & $1853 \mathrm{ab}$ & $7,8 \mathrm{abcd}$ & $5,6 a b c$ & 18,3 & b & 46,0 \\
\hline Talamanca & 2058 a & 5,8 & 5,4 bc & 23,8 a & & 47,5 \\
\hline
\end{tabular}

Cuadro 6. Producción de grano y otras variables agronómicas evaluadas en líneas de frijol Adzuki, en Río Frío, Heredia, Costa Rica, Noviembre 1985Febrero 1986.

\begin{tabular}{|c|c|c|c|c|c|}
\hline $\begin{array}{l}\text { Línea o } \\
\text { variedad }\end{array}$ & $\begin{array}{l}\text { Producción } \\
\text { (kg/ha) }\end{array}$ & Vainas/planta & Granos/vaina & \multicolumn{2}{|c|}{$\begin{array}{l}\text { Altura de } \\
\text { planta }(\mathrm{cm})\end{array}$} \\
\hline UCR 1 & $1900 \mathrm{~b}$ & 9,9 & $7,2 \quad b$ & 29,7 ef & \\
\hline UCR 2 & $2199 a b$ & 11,1 bc & $7,0 \quad b$ & $33,9 \mathrm{e}$ & \\
\hline UCR 3 & $2103 a b$ & 14,3 a & $6,4 \mathrm{~cd}$ & $33,7 \mathrm{e}$ & \\
\hline UCR 4 & 2527 a & 14,2 a & 7,9 a & 52,4 ab & \\
\hline UCR 5 & $2128 a b$ & $13,4 a b$ & $7,5 \mathrm{ab}$ & 78,3 & c \\
\hline UCR 6 & $2035 a b$ & $11,7 \mathrm{ab}$ & $6,5 \quad c$ & 29,2 & e \\
\hline UCR 7 & 2338 a & 17,4 a & 6,2 & 34,4 & e \\
\hline UCR 8 & $2090 \mathrm{ab}$ & 11,5 bc & $7,5 \mathrm{ab}$ & 32,5 & e \\
\hline UCR 9 & $1946 \mathrm{~b}$ & 11,7 bc & 6,3 & 29,8 & ef \\
\hline UCR 10 & $1813 \mathrm{bc}$ & $12,5 \quad b$ & $7,0 \quad b$ & 33,6 & e \\
\hline UCR 11 & 1920 b & 11,6 bc & 6,6 & 30,5 & e \\
\hline UCR 12 & $2040 a b$ & 11,6 bc & 6,7 & 26,0 & gh \\
\hline UCR 13 & $2193 a b$ & $13,6 a b$ & 5,9 & 28,6 & $\mathrm{fg}$ \\
\hline UCR 14 & $1864 b$ & $9,9 \quad \mathrm{c}$ & $7,2 \quad b$ & 28,6 & de \\
\hline México 80 & $1319 \mathrm{~cd}$ & 11,3 bc & $6,1 \mathrm{~cd}$ & 57,5 a & \\
\hline
\end{tabular}

\section{LITERATURA CITADA}

ANDRADE, E. 1975. Estudio de fechas de siembra de frijol rojo japonés. Agricultura técnica en México 3 (10): 393-396.

ARA YA, R; ZUMBADO, Z. 1983. Interacción de siete cultivares de frijol voluble con dos de maíz en asociaciones simultáneas. Boletín Técnico Estación Experimental Fabio Baudrit [C.R] 16 (2): 1-4.

CENTER FOR OVERSEA PEST-RESEARCH. 1981. Control in tropical pest research, Londres. Overseas Development Administration. 206 p.
CENTRO INTERNACIONAL DE AGRICULTURA TROPICAL. 1980. Diversidad genética de las especies cultivadas del género Phaseolus; guía de estudio para ser usada como complemento de la Unidad Audiotutorial sobre el mismo tema. Producción, Ospina, H. Asesoría Científica, Hidalgo, R; Song, L.; Gepts, P. Cali, Colombia. 52 p. (Serie 045B-69.02).

DELGADO, J.; ARAYA, R 1983. Cultivar es de Vigna spp. bajo tres épocas de siembra en Río Frío. Boletín Técnico Estación Experimental Pabio Baudrit [C.R] 16 (4): 1-6. 
DELGADO, J.; ARAYA, R.; CALDERON, S. 1984. Caracter ísticas organolépticas y porcentajes de proteína en cuatro especies de Vigna spp. Boletín Técnico Estación Experimental Fabio Baudrit [C.R] 17 (2): 1-8.

DUKE, J. 1981. Handbook of legumes of world economic importance. New York and London, Plenun Press. 56 p.

ESCALANTE, M.; ARAYA, R; MUSMANNI, M.; GONZALEZ, M. 1985. Evaluación de densidades de siembra en camote (Ipomoea batatas L.) asociado con Adzuki (Vigna angularis (Willd) Ohwi y Hoashi), soya (Glycine max L. Merr) y rabiza (Vigna unguiculata (L.) Walp). Boletín Técnico Estación Experimental Fabio Baudrit 18 (1): 1-7.

GONZALEZ, E.M.; ARAYA, R.; GONZALEZ, W. 1984. Evaluación de la asociación de dos ciclos vegetativos de frijol (Phaseolus vulgaris L.) con uno de maíz (Zea mays L.). Boletín Técnico Estación Experimental Fabio Baudrit [C.R.] 17 (4): 1-7.

HERNANDEZ, H.; ARAYA, R. 1986. Fertilización nitrofosfórica y distancias entre hileras en frijol Adzuki (Vigna angularis Willd Ohwi y Hoashi) en Río Frío. Boletín
Técnico Estación Experimental Fabio Baudrit [C.R] 19 (4): 1-18.

LAWN, R.J. 1983. Agronomic studies on Vigna spp. in south eastern Queeland III. Response to sowing arrangement. Aust. J. Agriculture Research 34: 505-515.

MATEO, J. 1961. Leguminosas de Grano. Barcelona, España. Salvat. p. 329-330.

ROBINSON, R.G. 1980. Registration of Minoka adzuki bean. Crop Science 20 (4): 549.

SACKS, F. 1977. A literature review of $P$. angularis. The adzuki bean. Economic Botany 31: 9-15

VIEIRA, C. 1967. O Feijoeiro comun: cultura, doencas e melhoramento. Vicosa, Brasil. Universidad e Rural de Minas Gerais. 220 p.

UNIVERSIDAD DE COSTA RICA. 1986. Desarrollo, adaptación y transferencia de tecnología para los sistemas de cultivo de asentamientos campesinos de Río Frío, Costa Rica. Informe de Progreso, Convenio UCR-CIID. San José, Costa Rica. pp. 43-56. 